\title{
Zero Power Criticality Benchmark Evaluation of the MSRE in WIMS
}

\author{
Charlie Constable $^{1}$, Dr Ben Lindley ${ }^{2}$, and Dr Geoff Parks ${ }^{1}$ \\ ${ }^{1}$ Cambridge University Engineering Department \\ Trumpington Street, Cambridge, CB2 1PZ, United Kingdom \\ ${ }^{2}$ Wood \\ Kings Point House, Queen Mother Square, Dorchester, DT1 3BW, United Kingdom \\ cc846@cam.ac.uk, ben.lindley@woodplc.com,gtp10@cam.ac.uk
}

\begin{abstract}
This paper discusses work done to benchmark the deterministic code WIMS [1] against the Monte Carlo code Serpent [2] and experiment. Comparison is made against the Molten Salt Reactor Experiment at Oak Ridge National Laboratory as well as a Serpent model produced at the University of California, Berkeley. Producing a model for an MSR is possible thanks to the development of the next version of WIMS, WIMS11. The structure of the WIMS model built is discussed, and the final predicted criticality value for the MSR is given. This compares favourably with the Serpent model; however, both codes predict values considerably different to those expected. Potential reasons for this are suggested. However, it is concluded that WIMS has successfully been benchmarked against the current state of the art. This provides confirmation that this is a valid approach for molten salt reactor research analysis.
\end{abstract}

KEYWORDS: Molten Salt Reactor, WIMS, Validation

\section{INTRODUCTION}

This paper describes a validation exercise for molten salt reactors (MSRs), utilising the deterministic reactor physics code WIMS11. MSRs are a proposed next generation fission reactor and, given their potential, it is important to develop codes to predict their behaviour, particularly as licensing cannot be obtained without a safety case supported by modelling. An exercise is described which aims to show the level of confidence in WIMS's predictions for MSRs, utilising experimental work from Oak Ridge National Laboratory. These WIMS calculations utilise the ENDF/BVII.0 nuclear data library. The benchmarking of the new WIMS model is then discussed, including an outline of the model and potential improvements that could be made in the future.

\section{PAPER BACKGROUND}

The motivation for this paper arises out of the development of the next (fourth) generation of nuclear reactors. Six systems were identified by an international forum to be researched in order to 
meet these goals. One of the proposed designs is the MSR, which offers improved safety features such as freeze plugs which drain the core at a critical temperature. Other advantages also exist, such as a relatively low amount of pipework compared to more traditional coolants, due to the good heat transfer properties of molten salt as a coolant. Finally, these reactors have the potential to breed new fissile material and utilise thorium fuel, which would allow far greater amounts of energy to be extracted from the earth's actinides inventory.

In this research, a software package called WIMS was used as a reactor modelling code. For a fuller discussion of WIMS' development, see [3]. WIMS is a modular neutronics prediction code, meaning that it allows the flux of neutrons in a nuclear reactor to be predicted. It is a deterministic code, meaning it is significantly faster computationally but at the cost of some accuracy. This is in comparison to other codes, such as Serpent or MONK which are discussed in this report. These codes use Monte Carlo statistics to create neutron flux profiles, which yields higher fidelity results but requires much greater computation. A new version of WIMS (WIMS11) is currently under development which can support modelling of MSRs, motivating its use in this project.

One point of concern as regards using neutronics codes to model MSRs is a lack of experimental results against which to test models. One of the few experiments carried out on this class of reactor was called the Molten Salt Reactor Experiment (MSRE). This experimental reactor was housed at the Oak Ridge National Laboratory in Tennessee between 1965 and 1969 [4]. One particular experiment of interest was the zero power criticality experiment. This was one of the initial experiments carried out upon the MSRE in 1965, but a fuller report was issued in 1968 describing the conclusions of this and other zero power physics experiments [5].

The reason the MSRE zero power criticality experiment has become of particular interest, however, is that an OECD/NEA benchmark has recently been developed for this experiment. In mid-2018 researchers at the University of California Berkeley (UCB) published a paper which described: the reactivity loss from introducing circulation of the fuel to the reactor, and the control rod worth. The UCB investigation indicated that their Serpent model was in reasonable agreement with the experimental values found by scientists at the MSRE [6]. This paper also indicated that a full benchmark specification was expected to be published in the International Reactor Physics Experiment Evaluation Project handbook by the summer of 2020. Therefore, a request was made to them to release their current version. They agreed to share a report, which was at the time unpublished [7] and has grown into the IRPhE benchmark [8]. The released UCB report describes the design of the model and the indicated criticality, the results of an uncertainty analysis of many different variables and the impact of certain changes to the model geometry on the reactor criticality. Therefore, it was decided to investigate the MSRE criticality experiment by replicating the UCB Serpent model as closely as possible.

\section{DESCRIPTION OF OAK RIDGE EXPERIMENT}

This section of the paper will outline the major aspects of the design of the MSRE as well as the method by which criticality was obtained. A more exact description of the MSRE can be found in [5]. 


\subsection{Geometry}

The first aspect to discuss is the geometry of the MSRE at the time of the first criticality experiment. For the purpose of benchmarking WIMS, the core of the reactor is the essential item of interest, and so the associated plant will not be described. A basic diagram of the reactor vessel is shown in Fig. 1.

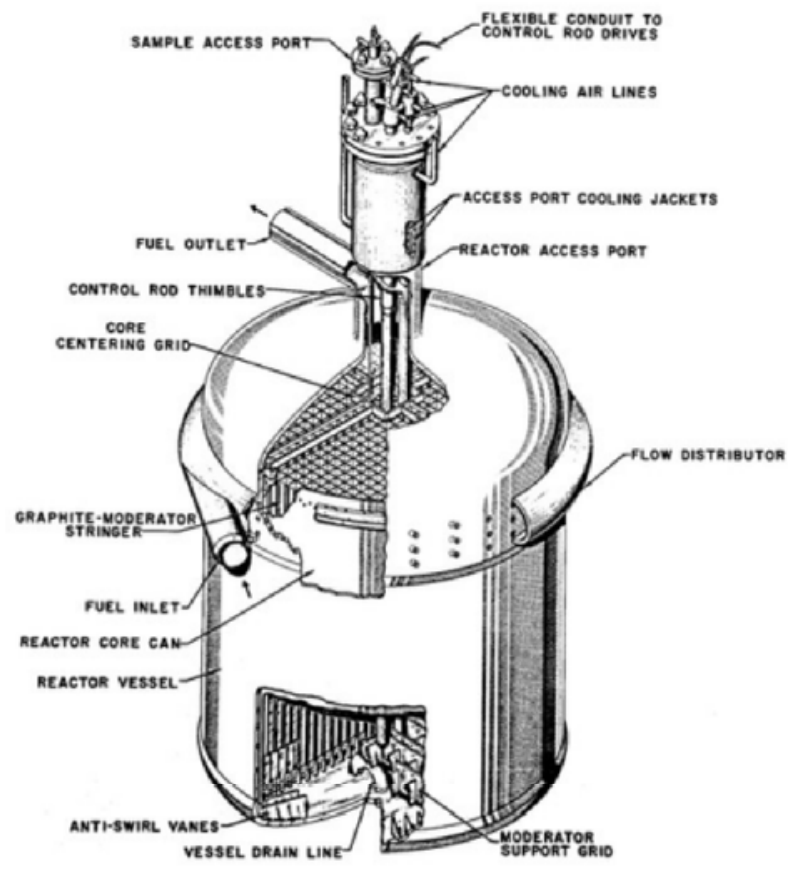

Figure 1: Diagram of MSRE core [6]

The most complex structure in the core of the MSRE is the graphite lattice. This lattice existed within the main region of the fuel salt so as to moderate neutrons produced by fissions within the fuel salt. The lattice was created by many long thin rods (stringers) of graphite which had small surrounding gaps when placed amongst other rods. This allowed the fuel salt to circulate around the graphite.

The structure of the graphite lattice was also different in the centre of the core where there was a control rod structure. This structure consisted of space for three control rods and a sample bed, which was intended to test the response of an experimental nickel alloy, INOR-8, to time exposed to a high temperature and radiation environment. One can see this structure in Fig. 2. The internal complexity in the control rod and sample basket structures is also clear in Fig. 2. A full description will not be given here for brevity, but can be found in reference [4].

\subsection{Conditions}

Within the core the salt could circulate freely around the graphite stringers. There are also both upper and lower tanks which hold a significant quantity of salt above and below the graphite lattice. 


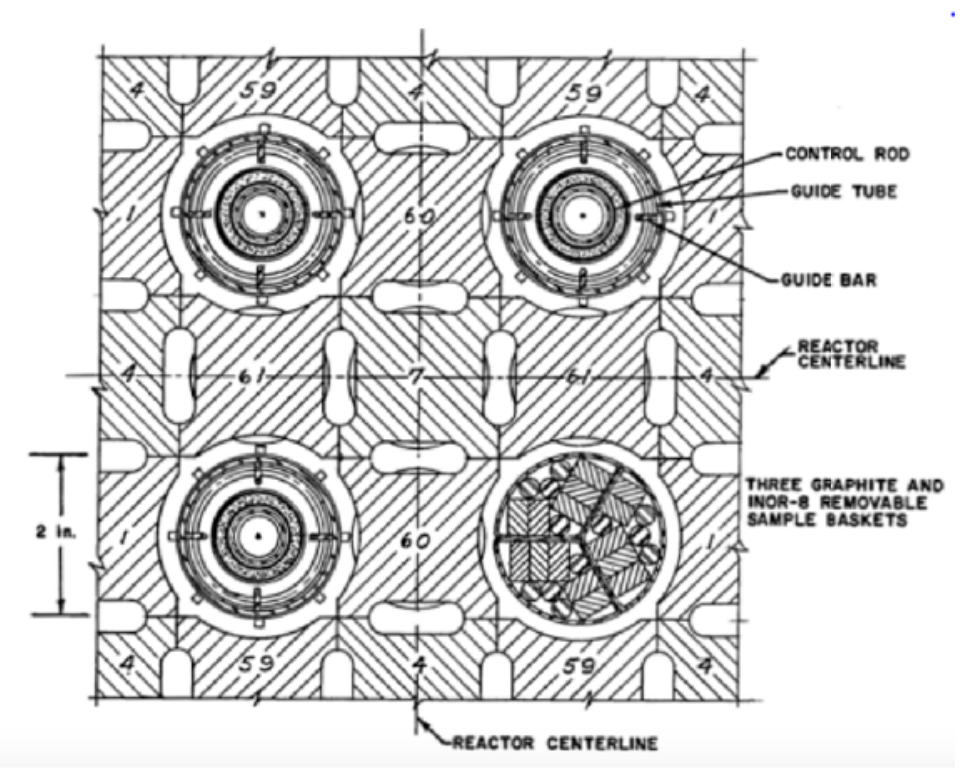

Figure 2: Control rod \& sample basket structure [5]

The spaces in the graphite lattice allow movement of salt between these tanks. Inlet and outlet pipes can be seen in Fig. 1.

Several layers of material exist around the core, which are important as they reflect a proportion of the neutrons which come into contact with them back into the core. The first of these layers, the core can, can be seen in Fig. 1, and contains the salt within the core. This is made of two layers of INOR-8, which allow salt to pass between them via a number of holes in the top section. There is then a cavity left, which is used to house the neutron instrument tube. The entire reactor vessel is held within a thermal shield, with four main layers. The innermost layer is made from high temperature insulating material called vermiculite. There are then two layers of stainless steel, which contain the thermal shield itself, consisting of small iron balls surrounded by water. The materials throughout the core were all maintained at a temperature of $911 \mathrm{~K}$ as the test was isothermal with no power draw or circulation.

\subsection{Process}

The zero power criticality experiment sought to determine the level of enrichment required in the fuel salt to just reach criticality. In order to do this, the reactor was initially filled with a salt containing only depleted uranium. The control rods were also inserted fully during initial fuelling of the reactor, before being fully withdrawn. In order to raise the criticality of the core, near pure ${ }^{235} \mathrm{U}$ (93\% enrichment) was dissolved into the salt. To ensure homogeneity of the salt, pumps were used during this process of adding highly enriched uranium, and a length of time of the order of a day was given between uranium additions. When predictions showed that the addition of the next batch of uranium would cause criticality, the control rods were inserted and the next batch added. The control rods were then slowly withdrawn until criticality was achieved. This explains the partial insertion of one of the rods in the MSRE, as a small amount of the worth of the rod was 
required to gain exact criticality. The circulation of the salt was stopped, and the reactor allowed to reach isothermal conditions before the final position of the control rod was found.

\section{DESCRIPTION OF WIMS MODEL}

In the first stage of the model, a large number of material definitions are created. A resonance shielding calculation is then performed on these different geometries using equivalence theory. A subgroup theory calculation is then carried out on the key nuclides (such as ${ }^{235} \mathrm{U}$ ). CACTUS is then used to define the geometries of different regions of the core (as can be seen in Fig. 3).

The next step is to call CACTUS again for each different geometry in order to calculate the criticality of that geometry in an infinite sea. This is done by repeating the defined block of geometry over an infinite area and using the method of characteristics to find the solution to the neutron transport equation. The materials that had their infinite criticality calculated in CACTUS are then homogenised. Consequently the geometry information within each complex region does not need to be maintained in the core calculation. MERLIN then uses the SP3 method to calculate the criticality of the core itself. An RZ model of the reactor is built, which requires cylindrical symmetry.

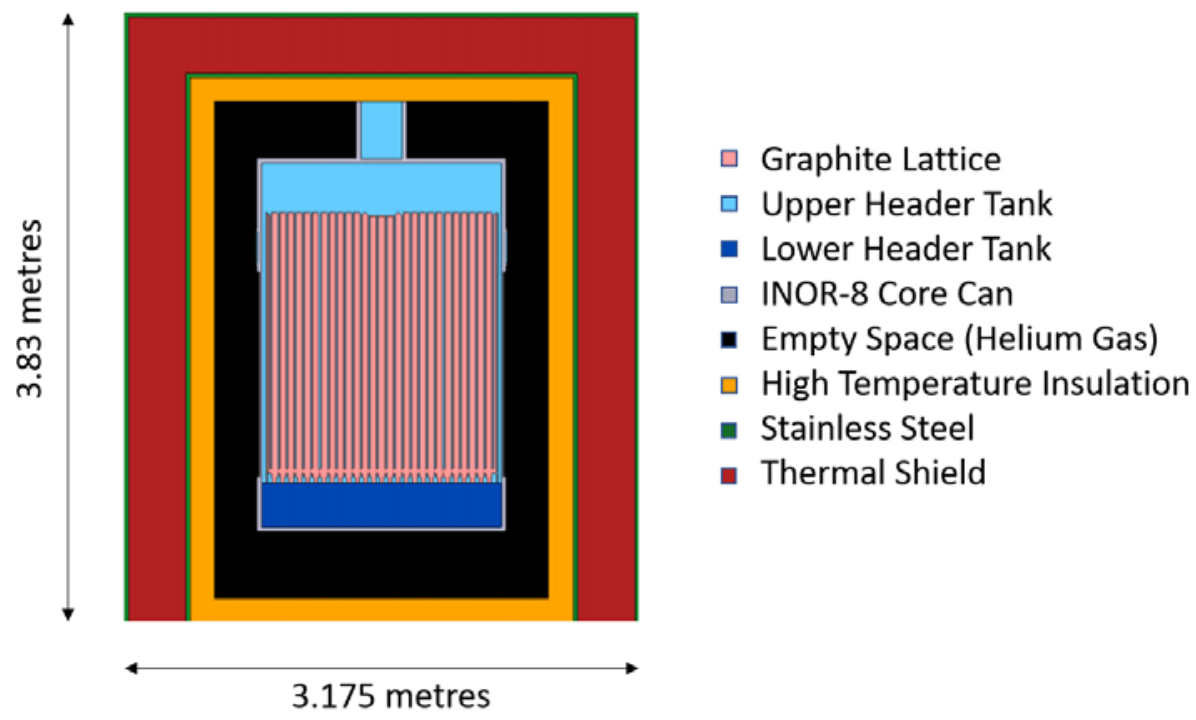

Figure 3: WIMS benchmark model, adapted from [7]

In order to benchmark WIMS's newly developed capability to model MSRs, the final position of the MSRE zero power criticality experiment was modelled. Hence, the salt composition was modelled as that at the point when all of the highly enriched uranium had been added to the salt. Similarly, the core was modelled as isothermal and with no artificial circulation of the salt. In the final WIMS model, the majority of the structures described in the previous section were built using CACTUS, and then these were passed to the MERLIN module, which carries out an SP3 RZ calculation of the flux in the core by knitting all of the CACTUS materials together. A number of simplifications were made when compared to the real MSRE reactor, but these will be discussed later in this section. 
Fig. 3 provides a diagrammatic representation of the modelled structure of the core. The central part of this figure gives a description of the different materials and their positioning through the core. The central pink region indicates the graphite lattice discussed in the previous section. The blue regions above and below it then indicate molten salt with different amounts of INOR-8 in the regions. The outer red and yellow regions describe the thermal shield and high temperature insulator respectively. In Fig. 4, the left-hand plan view cross section shows the geometry passed to CACTUS to create a homogenised material for the graphite lattice. The right-hand plan view cross section shows the control rod/sample bed geometry passed to CACTUS for the section where all three control rods are inserted. Section 3.3 described how, at criticality, the control rods were inserted by different amounts and this is reflected in the WIMS model by using different CACTUS geometries at each insertion level.

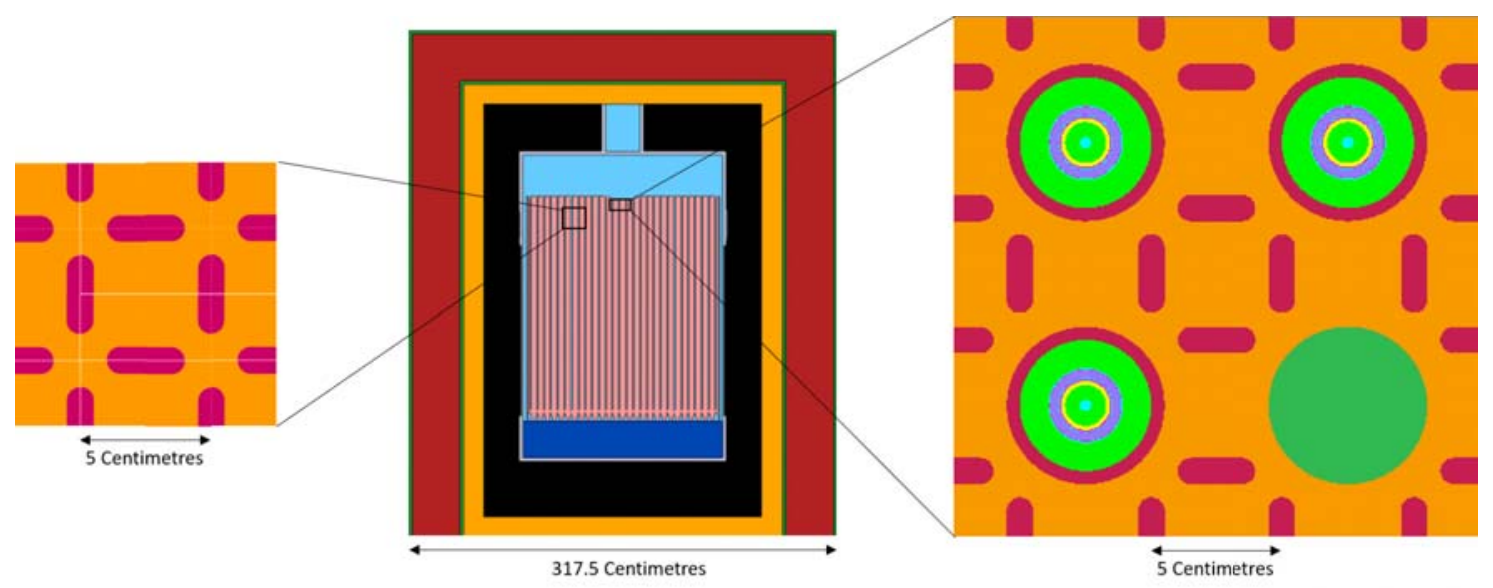

Figure 4: Examples of complex geometries passed to CACTUS

A 'super cell' calculation for the control rod sections was built for this WIMS model, which generates a more accurate flux shape in the control rods. This is to generate homogenised cross sections in a representative environment with an accurate calculation of the flux profile in the control rods. Therefore, a code was built which placed the control rod cell in a super cell, where it was surrounded by nine regions of equal area, each filled with the graphite lattice. An example of this geometry is shown in Fig. 5. A super cell was built for each control rod geometry in the core: none inserted, one inserted and all inserted.

The major simplifications made in the model are described here. Firstly, the reactor is converted to be axi-symmetric, which largely means that the inlet and outlet pipes and sensors which entered the core radially have been removed and replaced with the material they 'cut through'. The black region indicated in Fig. 3 was primarily an empty space containing instrumentation. The distributor, which was a ring of salt around the top of the graphite matrix, has been removed, due to difficulties in the RZ geometry. Similarly, the header tanks have been squared, to the same height as their original curved surface. 


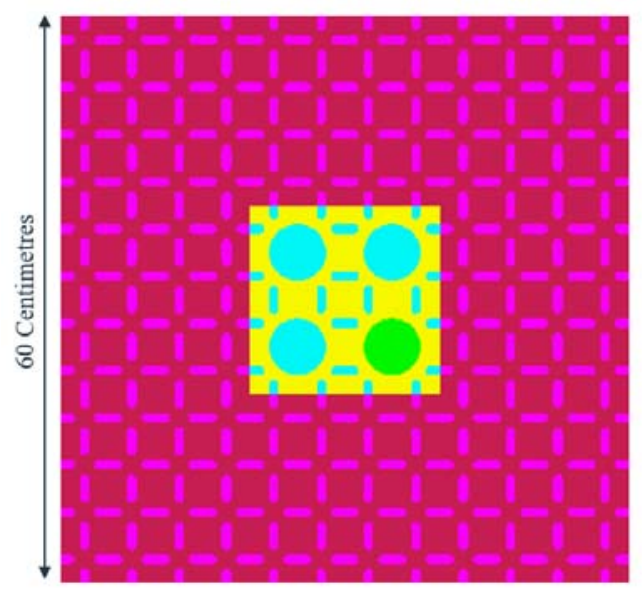

Figure 5: WIMS super cell geometric structure (no control rods inserted)

\section{RESULTS OF MODELLING}

The key value produced by the benchmark model which allows comparison is that of k-effective, which essentially describes the criticality of the core. As the experiment investigated just achieved criticality, the k-effective must have been exactly one (within a very small margin of error). As can be seen in Table I, the value of k-effective quoted for the MSRE is actually 0.997. The value quoted has been arrived at by applying investigating work done by UCB in [7]. In this work simplifications made to the core, such as making the core axisymmetric and removing the distributor, have had their effect on criticality evaluated. The results of this analysis have been used here to adapt the target value for the benchmark exercise, to account for major simplifications.

Table I: Benchmark modelling results

\begin{tabular}{|c|c|c|}
\hline Scheme & k-effective & Difference vs Experiment (\%) \\
\hline Experimental [7] & $0.997 \pm 0.00554$ & - \\
\hline Serpent (UCB) [8] & $1.0213 \pm 0.000095$ & 2.44 \\
\hline WIMS & 1.0229 & 2.60 \\
\hline
\end{tabular}

\section{DISCUSSION \& CONCLUSIONS}

The WIMS model is in very good agreement with the Serpent model, with a difference in keffective of just $160 \mathrm{pcm}$. As WIMS is a deterministic code while Serpent is a Monte Carlo code, this offers the appealing prospect of using WIMS to obtain good MSR modelling results quickly. Some of the difference in the results from the two codes could be due to the different nuclear data 
libraries used (Serpent used ENDF/VII.1 while WIMS used ENDF/BVII.0), though differences are anticipated to be minor. However, both models have a significant disparity with the experimental values: for an experimental reactor, a disparity of within one percent would generally be expected. This suggests that there is an unresolved issue with how the codes are modelling the MSRE.

There are some possible explanations for the disparity with experimental values. One of the most likely is that there are potential limitations or uncertainties in the nuclear data for MSRs, in particular for bound thermal scatter. This seems likely, considering this experimental reactor used some exotic materials which have not necessarily been tested independently. However, this seems unlikely to account for the full difference between models and experiment. It therefore seems plausible that some further difference between the MSRE and the software models exists. This discrepancy was described in the IRPhE benchmark as difficult to identify [8]. Nonetheless, as WIMS has closely matched the standard of the higher fidelity Monte Carlo code, it is reasonable to conclude that WIMS has been successfully benchmarked against current best practice for modelling an MSR with this calculational route. A validated deterministic code MSR benchmark model will make future sensitivity analysis studies of sources of experimental and calculational uncertainty easier.

\section{ACKNOWLEDGEMENTS}

The authors would like to thank Dr Max Fratoni and Dan Shen of UCB for their willingness to share at the time unpublished information. This has allowed concurrent working which has been greatly helpful to the authors.

\section{REFERENCES}

[1] B. Lindley et al., "Developments within the WIMS Reactor Physics Code for Whole Core Calculations", $M \& C 2017$, Jeju, Korea, 16-20 April (2017)

[2] M. Aufiero and M. Fratoni, "Testing Advanced Methods for Sensitivity/Uncertainty Analysis in the Monte Carlo Code Serpent", PHYSOR 2016, Sun Valley, USA, 1-6 May (2016)

[3] B. Lindley et al., "Current Status of the Reactor Physics Code WIMS and Recent Developments", Annals of Nuclear Energy, 102, pp. 148-157 (2017)

[4] R. C. Robertson, MSRE Design And Operations Report, Part I: Description Of Reactor Design, Oak Ridge National Laboratory, United States of America (1965)

[5] B. I. Prince, S. J. Ball, J. R. Engel, P. N. Haubenreich and T. W. Kerlin, Zero-Power Physics Experiments On The Molten-Salt Reactor Experiment, Oak Ridge National Laboratory, United States of America (1968)

[6] D. Shen, M. Fratoni, M. Aufiero, A. Bidaud, J. Powers and J. Ilas "Zero-Power Criticality Benchmark Evaluation of the Molten Salt Reactor Experiment", PHYSOR 2018, Cancun, Mexico, 22-26 April (2018)

[7] D. Shen and M. Fratoni, "Molten-Salt Reactor Experiment (MSRE) Zero-Power First Critical Experiment with ${ }^{235}$ U", unpublished, University of California, Berkeley, (2018)

[8] D. Shen and M. Fratoni, "Molten-Salt Reactor Experiment (MSRE) Zero-Power First Critical Experiment with ${ }^{235}$ U", in International Handbook of Evaluated Reactor Physics Benchmark Experiments, NEA/NSC/DOC(2006)01, Nuclear Energy Agency, Paris, France (2019) 